\title{
Genetic diversity and species diversity weaken plant-soil feedback-mediated coexistence
}

\author{
Lana Bolin ${ }^{1}$ and Jennifer Lau ${ }^{1}$ \\ ${ }^{1}$ Indiana University Bloomington
}

January 11, 2021

\begin{abstract}
Theory suggests that genetic diversity may influence species coexistence and that species diversity may influence genotype coexistence by altering competitive outcomes among species and genotypes, respectively. However, other coexistence mechanisms such as microbe-mediated plant-soil feedbacks (PSF), may also contribute. Interspecific PSF promotes species coexistence when plants grow better with heterospecific soil microbes than with conspecific microbes, and similarly, intraspecific PSF promotes genotype coexistence when plants grow better with heterogenotypic than with congenotypic microbes. Here, we tested whether genetic diversity influences the strength or direction of interspecific PSF and whether species diversity influences the strength or direction of intraspecific PSF. We found that genetic diversity reduced the capacity for interspecific PSF to promote species coexistence, and, for one study species, species diversity reduced the capacity for intraspecific PSF to promote genotype coexistence. These results suggest that genetic diversity and species diversity may weaken the ability of PSF to promote coexistence.
\end{abstract}

\section{Hosted file}

Bolin_PSF_EcolLett_resubmission2.pdf available at https://authorea.com/users/361861/articles/ 503515-genetic-diversity-and-species-diversity-weaken-plant-soil-feedback-mediatedcoexistence 